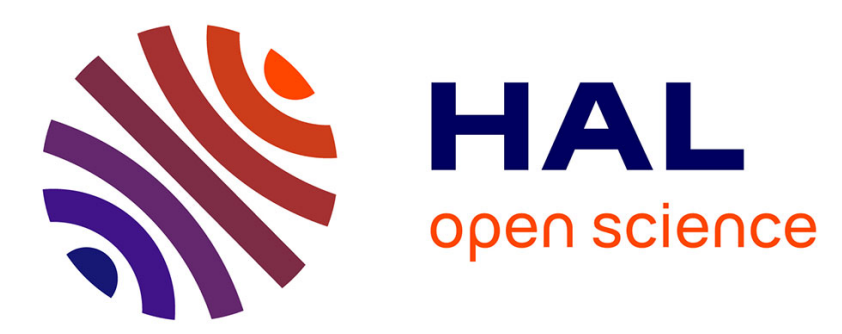

\title{
Geometrical Deviation Model of product throughout its life cycle
}

\author{
Dinh Son Nguyen, Frédéric Vignat, Daniel Brissaud
}

\section{To cite this version:}

Dinh Son Nguyen, Frédéric Vignat, Daniel Brissaud. Geometrical Deviation Model of product throughout its life cycle. International Journal of Manufacturing Research, 2011, 6 (3), pp.236-255. 10.1504/IJMR.2011.041128 . hal-00802498

\section{HAL Id: hal-00802498 https://hal.science/hal-00802498}

Submitted on 20 Mar 2013

HAL is a multi-disciplinary open access archive for the deposit and dissemination of scientific research documents, whether they are published or not. The documents may come from teaching and research institutions in France or abroad, or from public or private research centers.
L'archive ouverte pluridisciplinaire HAL, est destinée au dépôt et à la diffusion de documents scientifiques de niveau recherche, publiés ou non, émanant des établissements d'enseignement et de recherche français ou étrangers, des laboratoires publics ou privés. 


\title{
Geometrical Deviation Model of product throughout its life cycle
}

\section{Dinh Son Nguyen*, Frédéric Vignat and Daniel Brissaud}

\author{
G-SCOP Laboratory, \\ Grenoble Institute of Technology, \\ 46 avenue Félix Viallet, Grenoble 38031, France \\ E-mail: dinh-son.nguyen@g-scop.inpg.fr \\ E-mail: nguyen.dson@gmail.com \\ ${ }^{*}$ Corresponding author
}

\begin{abstract}
Nowadays, the requirements of customers concerning a product are more and more tight and high. The satisfaction of these, such as quality, reliability, robustness and cost, plays an important role in the context of global and concurrent economy. However, a product, during its life cycle, passes through manufacturing and assembly stages where geometrical deviations are generated and accumulated. These deviations that obviously have an influence on the performances of the product are not considered. Thus, a model based on the small displacement torsor that allows to model the geometrical deviations of the product is proposed in this paper.
\end{abstract}

[Received 18 March 2010; Revised 20 August 2010; Accepted 1 October 2010]

Keywords: life cycle engineering; manufacturing; product geometrical deviations.

Reference to this paper should be made as follows: Nguyen, D.S., Vignat, F. and Brissaud, D. (2011) 'Geometrical Deviation Model of product throughout its life cycle', Int. J. Manufacturing Research, Vol. 6, No. 3, pp.236-255.

\begin{abstract}
Biographical notes: Dinh Son Nguyen received his MS Degree in Industrial Engineering from Grenoble Institute of Technology in 2007. He is currently working as $\mathrm{PhD}$ student under the guidance of Professor Daniel Brissaud and Assistant Professor Frédéric Vignat in Integrated Design team of the Laboratory G-SCOP of Grenoble University. His research interests are modelling geometrical deviations of product, simulation of manufacturing processes and robust design methodology.
\end{abstract}

Frédéric Vignat obtained his $\mathrm{PhD}$ from Grenoble Institute of Technology in 2005. He is an Associate Professor at the University of Grenoble (Grenoble-INP). He is working in the Laboratory G-SCOP of Grenoble University in the area of Integrated Design. His research interests include manufacturing simulation, product tolerancing and life cycle engineering. He has published more than 20 papers in these areas in international journals and conferences. He mentored many Masters Theses and two PhD Theses.

Daniel Brissaud has been a Professor of Engineering Design and Eco-Design at the University of Grenoble (Grenoble-INP) since 1998. He is head of the cluster of research "Management and Organisation of Production Systems and Innovation" for the Rhône-Alpes area, member of the European Manufacturing 
and Innovation Research Association 'EMIRAcle', CIRP Fellow. He was head of the doctoral studies in Industrial organisations and production systems. His scientific interests are eco-design, environmental assessment, life cycle engineering, clean technologies, product-service systems design, integrated design and requirement engineering. He authored more than 60 papers in international journals and books and mentored $16 \mathrm{PhD}$ Theses.

\section{Introduction}

Due to the development of information technology, computers have now become a useful and effective tool to support product and process design engineering activities. Most of the product designers can easily create and manage a numerical model of the product by a variety of computer-aided tools, such as CAD/CAM, CAE, product data management and product life cycle management tools. The current computer-aided tools are actually effective to reduce the time to design and develop a new product. However, the numerical model of the product is a nominal representation of this product that does not take into account the variations generated all along the product life cycle such as geometrical deviations generated during the manufacturing and assembly stage of the product life cycle (see Figure 1). It can result in a designed product that does not fully meet the requirements of customers and users.

Figure 1 Closed-loop product life cycle (see online version for colours)

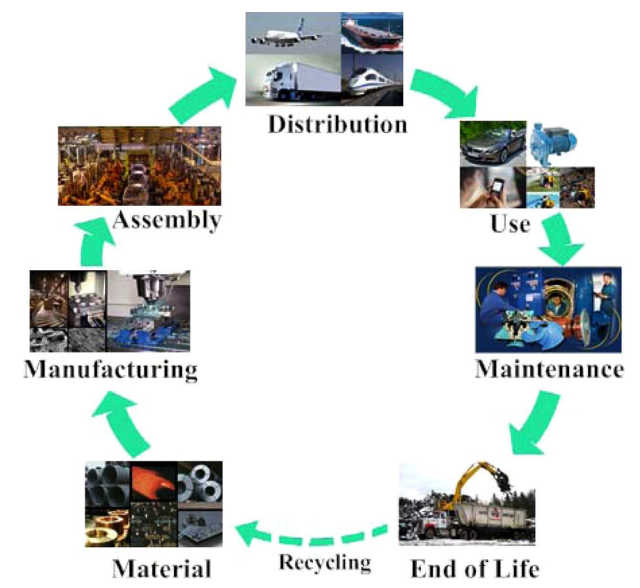

Each part making up the product is manufactured from raw material at the manufacturing stage. The quality of machined surfaces is modified in many ways such as roughness, surface hardness, and geometrical deviation surface. The geometrical deviations are generated on each surface of the part and accumulated over the successive setup of the manufacturing process. The parts with deviations are then assembled at the assembly stage. The deviations of the surfaces of each part, generated at manufacturing stage, influence the assemblability of the product. Later, the dimension and the quality of the final product are also affected by the accumulation of these deviations in each station of the assembly stage. Therefore, the geometry of the product is different from the nominal 
one at the end of these two production stages. The real performance of the product is then different from the designed one (nominal performance). The risk is then that the designed product does not satisfy the requirements of customers and users. In this case, the product-process design has to be considered as unsuitable or at least not to be robust.

To predict this variation of performance, two important issues have to be considered:

- How to model geometrical deviations of a product generated during manufacturing stage and accumulated over assembly stage of its life cycle?

- How to manage the causes and consequences of geometrical deviations at the design stage?

Some answers exist today in the academic research for each stage of the product life cycle separately. To find out a complete answer, it is necessary to develop a model of geometrical deviations of the product that is consistent with all the stages of the product life cycle. It has to allow the simulation of generation and accumulation of these deviations during manufacturing and assembly stages of the product life cycle and the integration of these deviations into the simulations of the product behaviour. The goal is to predict the performances of the product taking into account the geometrical deviations of the product.

This paper proposes a Geometrical Deviation Model (GDM) based on the Model Manufactured Part (MMP) (Vignat and Villeneuve, 2007). This model focuses on collecting the deviations of surfaces of a product generated during the manufacturing stage and accumulated over assembly stages of its life cycle because these deviations play an important role to influence performance of the product in use stage. Thus, the geometrical deviations generated in the other stages of the life cycle are not considered. However, further studies on the subject will be launched based on this GDM model.

\section{Literature reviews}

Some models exist to estimate the geometrical deviations of the part/product generated by the manufacturing/assembly processes in the manufacturing/assembly stages of its life cycle. These models stand for only one stage, manufacturing or assembly, but some of them could be used for both stages.

\subsection{Manufacturing stage}

Many researches work about machining error sources and link between manufacturing parameters such as workpiece materials, tooling, machining, cutting speed and cutting feed, and resulting errors such as surface roughness, surface deviation and surface hardness have been done. Ramesh et al. (2000a, 2000b) have studied the effect of cutting force and thermal effect on geometric deviation of the surfaces of the part. Thangavel and Selladurai (2008) have studied the influence of turning parameters, such as cutting speed, feed rate, depth of cut and tool nose radius on the surface roughness. The study was experimental and the mathematical model was obtained by using Response Surface Methodology (RSM). This model allows selecting quickly the process parameters to achieve the desired quality of machined surfaces. Sahoo et al. (2008) have studied the quality of milled surfaces affected by many parameters, such as spindle speed, depth of 
cut and feed rate and workpiece materials. They proposed a second-order mathematical models using RSM to allow the optimisation of the cutting condition to produce the best possible surface. Mohanasundararaju et al. (2008) worked on the quality of grinded surfaces and the influence of grinding parameters, such as wheel speed, work speed, traverse speed, in-feed, dress depth and dressing lead and developed a mathematical model for surface prediction. Ghasempoor et al. (2007) proposed a geometrical method to model surface finish in grinding. This model takes into account the stochastic nature of the grinding process and the kinematics of the chip generation. Given the grinding wheel specifications and cutting conditions, the model can produce the expected surface topography. The proposed model was evaluated by comparing the predictions with measured surface roughness obtained through grinding experiments. The results showed the predictions were consistent with the measurements, hence proving the effectiveness of the model. All these studies are experimental and deal with variations generated on one surface in one set-up. They have been done for the purpose of process optimisation and error compensation. However, it is not a main topic of this paper. We will only consider models that allow to model geometrical defects generated and accumulated over successive set-up.

Zhou et al. (2003) proposed a state space model to describe the dimensional variation propagation of multistage machining processes. When the workpiece passes through multiple stages, machining errors at each stage will be accumulated and transformed onto the workpiece. Differential motion vector, a concept from the robotics field, is used in this model as the state vector to represent the geometrical deviation of the workpiece. This vector is used as a state vector $x(k)$ to describe workpiece deviations at $k$ th stage. These deviations are accumulated and transformed onto the workpiece by previous stages $(1 \ldots k-1)$ of the multistage machining process. The set of state vectors $x(k)$ describe the workpiece deviations relative to nominal one resulting of the whole machining processes. This model provides a quantitative relationship between the fixture locator errors and the final workpiece geometrical error and has a great potential to be applied to fault diagnosis and process design evaluation for complicated machining processes.

Villeneuve et al. (2001) proposed a method to perform 3D manufacturing tolerancing for mechanical parts. The GDM of the part, the part-holder and the machining operations of a set-up in the manufacturing process, is based on the concept of SDT. The SDT concept, coming from metrology (Bourdet et al., 1996), has been used to model the variations coming from the positioning of the workpieces during the successive set-ups of the manufacturing process as well as from those due to the machining operations. Then, an MMP based on the SDT for simulating and storing the manufacturing defects in 3D has been developed by Vignat and Villeneuve (2007). It allows collecting the deviations generated during a virtual manufacturing process. The defects generated by a machining process are considered to be the result of two independent phenomena: positioning and machining, and the deviations are accumulated over the successive set-ups. The positioning deviation is the deviation of the part relative to the machine. The deviations of the manufactured surface relative to their nominal position in the MMP are expressed by the parameters of SDT.

Tichadou et al. (2005) proposed a graph representation of the manufacturing process. This graph allows to model the successive set-up based on the positioning surfaces, the machined surfaces This graph makes it possible to highlight the influential paths. They propose then two analysis methods. The first one uses an SDT model. The second 
one is based on the use of CAD software in which they model a manufacturing process with defects. They then virtually measure the realised part and check its conformity.

Dantan et al. (2008) proposed a geometrical specification model for the product life cycle. This model uses GeoSpelling model to manage geometrical variations along the product life cycle. However, it is only used to allow a complete and coherent tolerancing process along a life cycle and not to describe the geometrical deviations generated by manufacturing and assembly processes during the manufacturing and assembly stage of the product life cycle.

\subsection{Assembly stage}

A product is made up of parts assembled by the way of connections. Each part has to pass through the manufacturing stage where the geometrical deviations are generated. The product has to then continue through the assembly stage. Assembly stage of the product life cycle is an essential one of its life cycle, and it obviously brings its share of deviations to the product. Several models of dimensional variation propagation in the assembly stage have been proposed.

Ceglarek and Shi (1995) proposed a model of dimensional variation applied to sheet metal assembly. They apply their model to the automotive body assembly with the aim of making diagnosis and reducing the source of dimensional variability. Shiu et al. (1996) proposed a model of multi-station assembly process to diagnose automotive body dimensional deviations. This model is based on the design information coming from the CAD system and allows prediction of the system behaviour based on the in-line measurements of the final product. This model has only been applied to automotive sheet metal assembly. Hu and Stecke (2009) proposed a method based on the Stream of Variation (SoV) model to analyse the dimensional variation of the product during each station of assembly line and to compare different configurations (serial, parallel and hybrid line configuration) in the automotive body assembly. The variation in the final dimension is predicted by using worst case analysis, root sum squares, or Monte-Carlo simulation. This method also evaluates the productivity of the assembly line and its different configurations.

Mantripragada and Whitney (1999) proposed a model for mechanical assembly using State Transition Model approach. Two types of assemblies are addressed in this model: Type- 1 where the assembly process puts together parts that have their mating features fully defined by their respective manufacturing process prior to the final assembly and Type-2 where the process can incorporate in-process adjustments to redistribute variation. The total deviation in position and orientation is described by a $6 \times 1$ vector $\tilde{X}(k)$. State transition equation is then used to express relations between two processes at $k$ th assembly station:

Type 1:

$$
\tilde{X}(k+1)=A(k) \tilde{X}(k)+F(k) \tilde{w}(k)
$$

Type 2:

$$
\tilde{X}(k+1)=A(k) \tilde{X}(k)+B(k) \tilde{U}(k)+F(k) \tilde{w}(k)
$$


where

$\tilde{w}(k): 6 \times 1$ vector describing the variation associated with the part being assembled at the $k$ th assembly station, expressed in local part coordinates.

$F(k): 6 \times 6$ matrix that transforms the variation associated with the incoming part at the $k$ th assembly station from part $k$ 's coordinate frame to the base coordinate frame of the Data Flow Chain (DFC).

$A(k), B(k)$ : identity matrix.

$\tilde{U}(k): 6 \times 1$ vector describing the property of the absorption zone modelling the contact between fixture and part.

Finally, all dimensional variations from successive assembly station are accumulated into vector $\tilde{X}(k)$ at the final assembly station. However, this model for the mechanical assembly only simulates the assembly process of perfect parts. The geometrical deviations generated during manufacturing stage of each part are not taken into account in this model.

Huang et al. (2007) proposed a Stream-of-Variation Model for 3D rigid assemblies' dimensional variation propagation analysis in multi-station processes. This model is also based on State Space Model approach. The deviations accumulated at $i$ th assembly station are described by a vector $X(i) \in R^{n_{t} \times 1}$. The state space model of a multistage assembly process is represented by equations (3).

$$
\left\{\begin{array}{l}
X(i)=A(i-1) X(i-1)+B(i) U(i)+W(i) \\
Y(i)=C(i) X(i)+V(i)
\end{array}\right.
$$

where $U(i) \in R^{m(i) \times 1}$ is the fixture/part deviation contribution from station $i, Y(i) \in R^{q(i) \times 1}$ is the measurement obtained on station $i, W(i), V(i)$ are mutually independent noise and $A(i), B(i)$ and $C(i)$ are transformation matrix.

Thiebaut (2001) proposed a model to allow analysing the position variation of the part in the assembly based on the concept of SDT. The positioning variation of the part relative to its nominal position in the global coordinate system is expressed by equation (4).

$$
D(A / R)=E\left(A / S_{A}\right)+T\left(S_{A} / S_{B}\right)+E\left(S_{A} / B\right)+D(B / R)
$$

where

$D(A / R)$ is the variation of part $A$ relative to its nominal position in the global coordinate system.

$E\left(A / S_{A}\right)$ is the variation of surface $S_{A}$ of the part $A$ relative to its nominal position.

$T\left(S_{A} / S_{B}\right)$ is the variation of the link between the surface $S_{A}$ of the part $A$ and the surface $S_{B}$ of the part $B$.

$E\left(S_{A} / B\right)$ is the variation of surface $S_{B}$ of the part $B$ relative to its nominal position.

$D(B / R)$ is the variation of part $B$ relative to its initial nominal position in the global coordinate system. 
A linear system of equations is created along all the connections between part $A$ and part $B$. The positioning variation of part $A$ is determined by the resolution of the linear system of equations with the Gauss-Pivot method.

The main principle of the models proposed by Mantripragada and Whitney (1999), Huang et al. (2007) and Thiebaut (2001) is to model the variation of the part in each stage along the assembly process. However, they do not take into account the geometrical deviations of the surfaces of the parts linking to any parameters of the manufacturing process. Furthermore, the parameters of the SDT in MMP (Vignat and Villeneuve, 2007) link to the manufacturing process and can be measured as proposed by Tichadou et al. (2007). That is the reason why we propose, in this paper, a model using the SDT to model geometrical deviations generated from the manufacturing stage and accumulated in the assembly stage.

\section{GDM of product}

Some models of geometrical deviations for manufacturing and assembly process simulation already exist and are presented above. However, these models are not consistent with the whole life cycle of the product. Thus, a model of geometrical deviations suitable with all stages of the product life cycle is necessary.

\subsection{Small Displacement Torsors (SDT)}

The concept of SDT was developed in the 1970s by Bourdet and Clement (1976) to solve the problem of fitting a model of geometrical surface with a cloud of points in three-dimensional metrology. This concept is based on rigid body motions. An SDT $T$ at a point $\mathrm{O}$ in the Cartesian coordinate system $O X Y Z$ is described by rotational vector $R$ and translational vector $D$, as shown in equation (5).

$$
T=\{R \quad D\}_{\{O, X Y Z\}} .
$$

A surface deviation torsor is an SDT describing the deviation between an associated surface and a nominal one. The associated surface is an ideal surface associated to the real surface using a minimum distance criterion such as the least square. For example, the deviation torsor of the associated plane relative to its nominal position (see Figure 2) is described by the SDT $T_{\text {Surface }}$ at a point $\mathrm{O}$ in the local coordinate system $O X Y Z$, as given in equation (6).

$$
T_{\text {Surface }}=\left\{\begin{array}{cc}
r x & 0 \\
r y & 0 \\
0 & t z
\end{array}\right\}_{\{O, X Y Z\}}
$$

where $r x, r y$ and $t z$ are rotational and translational components regarding $X, Y$ and $Z$ axis, respectively. The plane, in this case, has three degrees of freedom, so three positioning deviations of the plane are invariant (i.e., cannot be measured due to the surface class) relative to their nominal position and their values are arbitrarily fixed. Thus, the 0 value is chosen to hide the notion of invariance. 
Figure 2 Deviation torsor of a plane (see online version for colours)

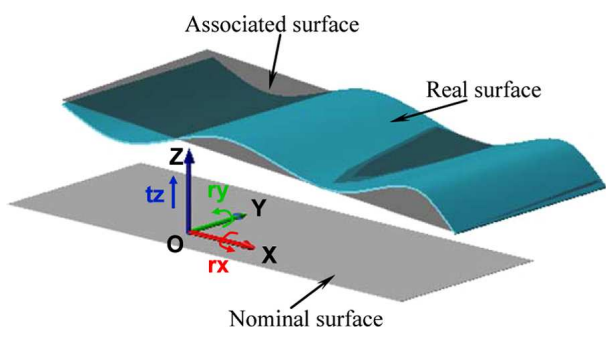

Source: Kamali Nejad et al. (2009)

A link deviation torsor expresses a deviation of the relative position of two surfaces coming from different parts. This deviation is expressed compared with their nominal relative position. The link deviation torsor is associated to each couple of surfaces of a mechanism that build up a connection. It then only concerns two surfaces that belong to different parts. The components of the link deviation torsor are divided into determined components $(l r, l t)$ and undetermined ones $(U l r, U l t)$. These components $(U l r, U l t)$ of the link deviation torsor express the relative mobility degrees of the two connected surfaces. In other words, they are the degrees of freedom of the connection. The components of a link deviation torsor of some elementary connections are expressed in Table 1.

Table 1 Elementary connections and related link torsor

\begin{tabular}{|c|c|c|}
\hline Elementary connection & Coordinate system & Link torsor \\
\hline Punctual & & $\left\{\begin{array}{cc}\text { Ulrx } & \text { Ultx } \\
\text { Ulry } & \text { Ulty } \\
\text { Ulrz } & \text { ltz }\end{array}\right\}_{\{O, X Y Z\}}$ \\
\hline Plane-plane & & $\left\{\begin{array}{cc}\text { lrx } & \text { Ultx } \\
\text { lry } & \text { Ulty } \\
\text { Ulrz } & \text { ltz }\end{array}\right\}_{\{O, X Y Z\}}$ \\
\hline Cylinder-cylinder & & $\left\{\begin{array}{cc}\text { lrx } & \text { ltx } \\
\text { lry } & \text { lty } \\
\text { Ulrz } & \text { Ultz }\end{array}\right\}_{\{O, X Y Z\}}$ \\
\hline
\end{tabular}

Source: Kamali Nejad et al. (2009)

A part deviation torsor is an SDT associated to each part. It describes the positioning deviation of the nominal part associated to the real part relative to its nominal position in the nominal assembly (see Figure 3). It expresses the assembly deviation due to part surfaces' deviations and gap among part surfaces.

The part deviation torsor expresses the position of the part in the $3 \mathrm{D}$ space and thus contains 6 parameters ( 3 rotations and 3 translations), as given in equation (7).

$$
T_{\text {Part }}=\left\{\begin{array}{cc}
r x & t x \\
r y & t y \\
r z & t z
\end{array}\right\}_{\{O, X Y Z\}} .
$$


To realise torsor operations (addition or subtraction), each torsor must be expressed at the same point and in the same coordinate system. Thus, it is necessary to transform the torsor from a point to another point or from a coordinate system to another coordinate system.

Figure 3 Deviation of real part and nominal part (see online version for colours)

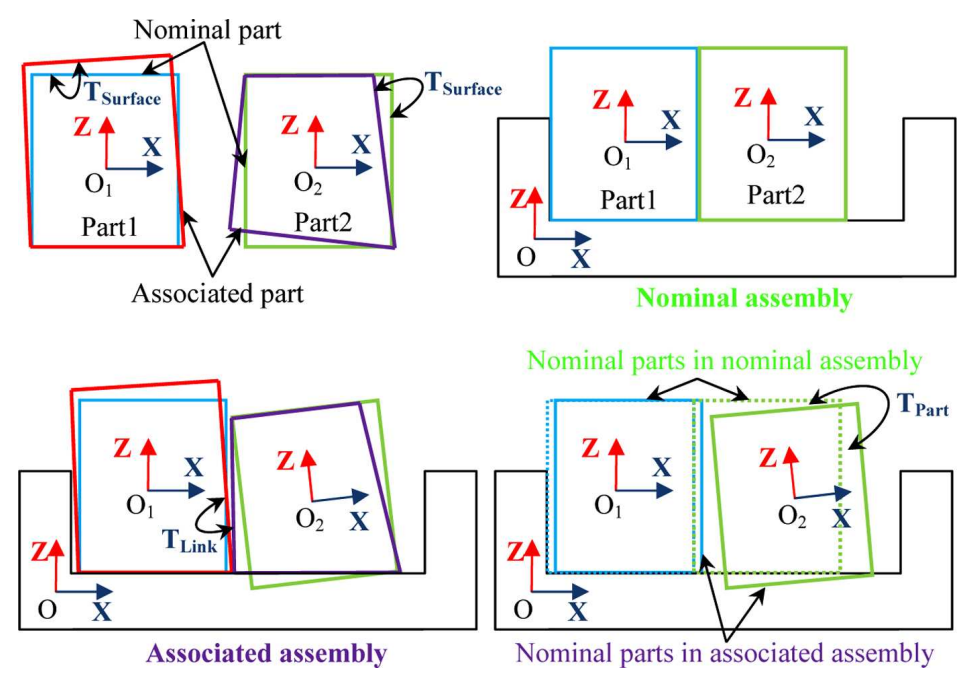

\subsection{Model of Manufactured Part (MMP)}

The GDM for the manufacturing stage is based on the MMP (Vignat and Villeneuve, 2007). It describes the geometrical deviations generated and accumulated for each surface of the manufactured part relative to its nominal position. The geometrical deviations of surface $j$ of manufactured part $i$, manufactured in set-up $S j$ relative to its nominal position can be expressed by equation (8).

$$
T_{P^{i}, P_{j}^{i}}=-T_{S j, P^{i}}+T_{S j, P_{j}^{i}}
$$

$T_{S j, P_{j}^{i}}$ is an SDT modelling the machining deviation of the machined surface $j$ realised in set-up $S j$. This deviation is expressed relative to the nominal machine. This torsor merges deviations of the surface swept by the tool and cutting local deformations.

$T_{S j, P^{i}}$ is a part deviation torsor modelling the positioning deviation of workpiece $i$ in set-up Sj. This deviation is function of the MMP surfaces deviation generated by the previous set-ups, the fixture surfaces' deviations and the links between fixture and part surfaces. This SDT is the result of the sum of the three mentioned deviations and is calculated by equation (9).

$$
T_{S j, P^{i}}=-T_{P^{i}, P_{m}^{i}}+T_{S j, H i S j}+T_{H i S j, P_{m}^{i}}
$$

$T_{P^{i}, P_{n}^{i}}$ is a surface deviation torsor modelling the deviation of surface $m$ of part $i$, which has been realised in previous set-up.

$T_{H i S j, P_{n}^{i}}$ is a link deviation torsor modelling a characteristic link between surface $m$ of part $i$ and the corresponding surface of the fixture in phase $S j$. 
$T_{S j, H i S j}$ is an SDT modelling the deviations of the fixture surfaces. It is a combination of fixture surface deviations and fixture deviation relative to their nominal position in set-up $S j$. It is calculated by equation (10).

$$
T_{S j, H i S j}=T_{S j, H}+T_{H, H i S j} .
$$

$T_{S j, H}$ is a part deviation torsor modelling the positioning deviations of the fixture relative to its nominal position in the machine.

$T_{H, H i S j}$ is a surface deviation torsor modelling the deviations of surface $i$ of the fixture relative to its nominal position in phase $S j$.

For a specific connection, undetermined components $(U l r, U l t)$ of the link deviation torsor $T_{H i S, P_{m}^{i}}$ remain in the positioning deviation torsor $T_{S i, P^{i}}$. They express the degrees of freedom of this elementary connection. However, the workpiece is completely positioned on the part-holder in set-up $S j$ by a set of elementary connection. This result is a fully constrained or overconstrained assembly. The mathematic expression of the unique position of the workpiece in set-up $S j$ is obtained by elimination of the undetermined components of the link deviation torsors. To eliminate these components (Ulr, Ult) of the link deviation torsor $T_{H i S j, P_{m}^{i}}$, the positioning deviation torsor $T_{S j, P^{i}}$ is calculated by another connection between surface $n$ of workpiece $i$ and surface $k$ of the part-holder in set-up $S j$.

$$
T_{S j, P^{i}}=-T_{P^{i}, P_{n}^{i}}+T_{S j, H K S j}+T_{H K S j, P_{n}^{i}} .
$$

The torsor $T_{S j, P^{i}}$ coming from equation (9) is equal to the torsor $T_{S j, P^{i}}$ coming from equation (11). The 'unification' method proposed by Villeneuve and Vignat (2005) allows to establish relations between the undetermined components $(U l r, U l t)$ and the determined ones $(l r, l t)$. These relations are obtained by Gauss Pivot method. The elimination of the undetermined components is then realised by replacing them, using these relations, in the positioning deviation torsor $T_{S j, P^{i}}$.

Finally, the whole deviations generated and accumulated by the manufacturing process are collected in the MMP and the deviations of each surface $j$ of the manufactured part $i$ are expressed by SDT $T_{P^{i}, p^{i}}$.

The variation of the parameters of the MMP is limited and this limitation is managed by constraints. The constraints on the part-holder surfaces' deviations $(\mathrm{CH})$ are relative to its quality (precision of its surface). The constraints on the machining deviations (CM) are relative to the machine capabilities.

The constraints on the links between part and part-holder surfaces (CHP) represent assembly rules. They are the constraints on the determined components $(l r, l t)$ of the link deviation torsor. The constraints depend on the type of connection (floating or slipping). The first constraint to satisfy is non-penetration conditions. In other words, workpiece material must not penetrate fixture material.

In the case of slipping contact, it is necessary to bring the surfaces close to each other. To do that, a positioning function is defined. This function expresses the displacement of a point of the workpiece along a direction relevant to the contact. The function increases when the surfaces of the connection move closer.

For example, plane/plane connection, this positioning function expresses the displacement of a point of the workpiece along a direction that is normal to the plane and in a direction that brings the two planes closer. 
Thus, for this kind of contact, the displacement function has to be maximised, respecting the non-penetration conditions between workpiece and part-holder (see Table 2).

Table 2 Non-penetration conditions and positioning functions of elementary connections

\begin{tabular}{|c|c|c|c|c|c|}
\hline $\begin{array}{l}\text { Elementary } \\
\text { connection }\end{array}$ & $\begin{array}{c}\text { Coordinate } \\
\text { system }\end{array}$ & Link torsor & & Non-penetration conditions & $\begin{array}{l}\text { Positioning } \\
\text { function }\end{array}$ \\
\hline Punctual & & $\left\{\begin{array}{cc}\text { Ulrx } & \text { Ultx } \\
\text { Ulry } & \text { Ulty } \\
\text { Ulrz } & \text { ltz }\end{array}\right\}$ & $\left\{\begin{array}{l}\{O, X Y Z\} \\
\end{array}\right.$ & $l t z \geq 0$ & $-l t z$ \\
\hline Plane-plane & & $\left\{\begin{array}{cc}\text { lrx } & \text { Ultx } \\
\text { lry } & \text { Ulty } \\
\text { Ulrz } & \text { ltz }\end{array}\right\}$ & $\{O, X Y Z\}$ & $\begin{array}{l}-\frac{b}{2} l r x-\frac{a}{2} l r y-l t z \geq 0 \\
-\frac{b}{2} l r x+\frac{a}{2} l r y-l t z \geq 0 \\
\frac{b}{2} l r x-\frac{a}{2} l r y-l t z \geq 0 \\
\frac{b}{2} l r x+\frac{a}{2} l r y-l t z \geq 0\end{array}$ & $-l t z$ \\
\hline $\begin{array}{l}\text { Cylinder- } \\
\text { cylinder }\end{array}$ & 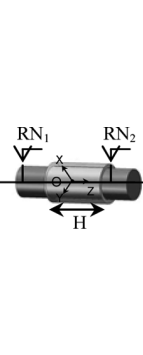 & $\left\{\begin{array}{cc}l r x & \text { ltx } \\
\text { lry } & \text { lty } \\
\text { Ulrz } & \text { Ultz }\end{array}\right\}$ & $\{O, X Y Z\}$ & $\begin{array}{l}R N_{2}-R N_{1}+r_{2}-r_{1}+\sqrt{(l t}+ \\
R N_{2}-R N_{1}+r_{2}-r_{1}+\sqrt{(l t)}+\end{array}$ & $-R N_{2}$ \\
\hline
\end{tabular}

For floating contact, the determined components need only to comply with the non-penetration condition between the workpiece and the part-holder.

\subsection{Model of Assembled Part (MAP)}

Then the MMPs are assembled to make up the product with deviations. The assembly model is called MAP and is based on the gap torsor concept proposed by Bourdet et al. (1996). The geometrical deviations of surface $j$ of part $i$ relative to the global coordinate system is expressed by equation (12).

$$
T_{P, P_{j}^{i}}=T_{P, P^{i}}+T_{P^{i}, P_{j}^{i}}
$$

where $T_{P^{i}, P_{i}^{i}}$ is a surface deviation torsor modelling the deviations of surface $j$ of part $i$ relative to its nominal position in the local coordinate system of the part $i$, coming from the manufacturing stage. It has already been presented above as a component of MMP $i$.

$T_{P, P^{i}}$ is a part deviation torsor modelling the positioning deviations of part $i$ relative to its nominal position in the global coordinate system of the product. It not only depends on the deviations of the surfaces of part $i$ but also the links between the surfaces of part $i$ 
and the surfaces of any other connected part $k$. The deviation of the concerned surfaces of part $k$ and the position of part $k$ is relative to the global coordinate system of the product. The torsor $T_{P, P^{i}}$ is determined by equation (13) for an elementary connection between part $i$ and part $k$.

$$
T_{P, P^{i}}=T_{P, P^{k}}+T_{P^{k}, P_{n}^{k}}+T_{P_{n}^{k}, P_{m}^{i}}-T_{P^{i}, P_{m}^{i}}
$$

where $T_{P_{n}^{k}, P_{m}^{i}}$ is a link deviation torsor between surface $m$ of MMP $i$ and surface $n$ of MMP $k$.

$T_{P, P^{k}}$ is a part deviation torsor modelling the positioning deviations of part $k$ (a sub assembled part coming from the previous set-up of the assembly process) relative to its nominal position in the global coordinate system of the product.

The position of part $i$ depends on several elementary connections with the part $k$ and the other parts connected with part $i$. Thus, the positioning deviation for each elementary connection is expressed by equations (14)-(16):

Link 1:

$$
T_{P, P^{i}}=T_{P, P^{k}}+T_{P^{k}, P_{m}^{k}}+T_{P_{m}^{k}, P_{n}^{i}}-T_{P^{i}, P_{n}^{i}}
$$

Link 2:

$$
T_{P, P^{i}}=T_{P, P^{k}}+T_{P^{k}, P_{q}^{k}}+T_{P_{q}^{k}, P_{p}^{i}}-T_{P^{i}, P_{p}^{i}}
$$

Link $n$ :

$$
T_{P, P^{i}}=T_{P, P^{k}}+T_{P^{k}, P_{z}^{k}}+T_{P_{z}^{k}, P_{y}^{i}}-T_{P^{i}, P_{y}^{i}}
$$

And, as far as the part is rigid and can have only one position, it is possible to write a linear system of equations (17).

$$
\left\{\begin{array}{l}
\text { Link } 1=\operatorname{Link} 2 \\
\operatorname{Link} 1=\operatorname{Link} 3 \\
\cdots \\
\operatorname{Link}(n-1)=\operatorname{Link} n
\end{array} .\right.
$$

The resolution of this system by the gauss pivot method is similar to the resolution presented for MMP positioning. It is also called unification and eliminates the undetermined components of each link deviation torsor between the part $i$ and the part $k$ and the position of the part $i T_{P, P^{i}}$ can be completely determined function of determined links and surfaces deviations parameters. Finally, the geometrical deviations of surface $j$ of the part $i$ in the global coordinate system can be calculated by the equation (12).

In conclusion, the whole geometrical deviations of all surfaces of the product relative to their nominal positions in the global coordinate system will be collected by SDT $T_{P, P_{j}^{i}}$. The variation parameters of the SDT are limited by the manufacturing constraints $(\mathrm{CH}, \mathrm{CM}, \mathrm{CHP})$ and assembly constraints (CA). The geometrical deviations of 
the product generated by the manufacturing and assembly processes during the manufacturing and assembly stages of its life cycle have been calculated and stored.

\section{Case study}

\subsection{Centrifugal pump design}

In this paper, a GDM for product life cycle engineering has been presented. To demonstrate this method, an example of centrifugal pump design is developed. The CAD model of the centrifugal pump is represented Figure 4.

Figure 4 The parts of designed centrifugal pump (see online version for colours)

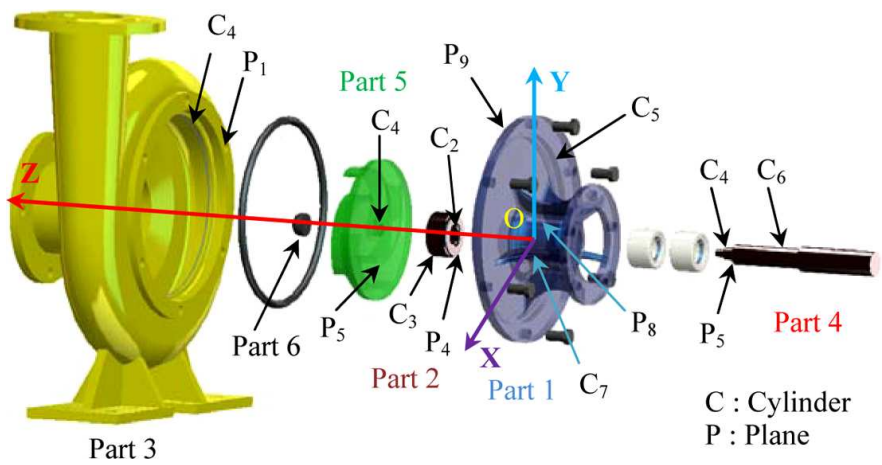

\subsection{Model Manufactured Part of the centrifugal pump}

To manufacture the pump, a manufacturing process, an assembly process and the associated resources are proposed. As a result, the GDM of this centrifugal pump from manufacturing and assembly stages of its life cycle is generated by the method presented in Chapter 3.

For example, the shaft (part 4) of the pump is realised by a turning process on a lathe machine (see Figure 5), according to the process plan described in Table 3.

Figure 5 Model of manufactured shaft of the pump (see online version for colours)

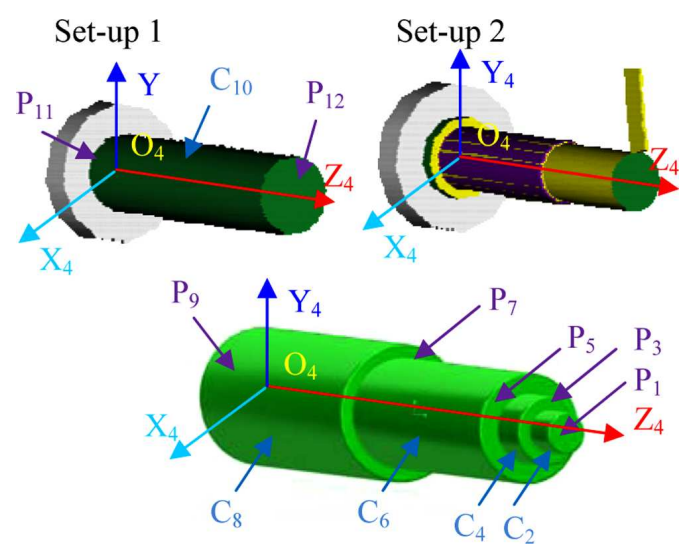


Table 3 Turning process

\begin{tabular}{ll}
\hline Set-up 1 & \\
\hline Positioning & Cylinder on 10 \\
Machining & Plane on 11 \\
\hline Set-up 2 & Surface $8,9,12$ \\
\hline Positioning & \\
& Cylinder on 8 \\
Machining & Plane on 9 \\
\hline
\end{tabular}

The geometrical deviations of each surface $j$ of the shaft are expressed by the SDT $T_{P^{4}, P_{j}^{4}}$. To calculate this torsor, it is necessary to determine the positioning deviation of workpiece in set-up 1 and 2.

For example, the positioning deviation of workpiece in set-up 1 is determined by the connection between the surface $C_{10}$ and the part-holder surface. It is expressed by equation (18).

$$
T_{S 1, P^{4}}=-T_{P^{4}, P_{10}^{4}}+T_{S 1, H 10 S 1}+T_{H 10 S 1, P_{10}^{4}}
$$

where, $T_{p^{4} P^{4}}$ is a surface deviation torsor modelling the deviations of cylinder $C_{10}$ of the workpiece. It comes from previous set-up 0 where the rough part has been manufactured. It is described at the centre point $\mathrm{O}_{\mathrm{C} 10}$ of the cylinder $C_{10}$ in the local coordinate system $X_{4} Y_{4} Z_{4}$ of part 4 by equation (19).

$$
T_{P^{4}, P_{10}^{4}}=\left\{\begin{array}{cc}
r x_{4,10} & t x_{4,10} \\
r y_{4,10} & t y_{4,10} \\
0 & 0
\end{array}\right\}_{\left\{O_{C 10}, X_{4} Y_{4} Z_{4}\right\}} .
$$

$T_{S 1, H 10 S 1}$ is an SDT modelling the deviations of the part-holder surface $H_{10}$. It is described at the point $\mathrm{O}_{\mathrm{C} 10 \mathrm{H}}$ of the part-holder in the local coordinate system $X_{4} Y_{4} Z_{4}$ by equation (20).

$$
T_{S 1, H 10 S 1}=\left\{\begin{array}{cc}
r x_{4,10 S 1} & t x_{4,10 S 1} \\
r y_{4,10 S 1} & t y_{4,10 S 1} \\
0 & 0
\end{array}\right\}_{\left\{O_{C 10 H}, X_{4} Y_{4} Z_{4}\right\}} .
$$

$T_{H 10 S 1, P_{10}^{4}}$ is a link deviation torsor modelling characteristic link between cylinder $C_{10}$ of the workpiece and the surface $H_{10}$ of the part-holder. It is expressed at the point $\mathrm{O}_{\mathrm{C} 10 \mathrm{~L}}$ in the local coordinate system $X_{4} Y_{4} Z_{4}$ by equation (21).

$$
T_{H 10 S 1, P_{10}^{4}}=\left\{\begin{array}{cc}
l r x_{4,10 S 1} & l t x_{4,10 S 1} \\
l r y_{4,10 S 1} & l t y_{4,10 S 1} \\
U l r z_{4,10 S 1} & U l t z_{4,10 S 1}
\end{array}\right\}_{\left\{O_{C 10 L}, X_{4} Y_{4} Z_{4}\right\}} .
$$

The positioning deviation of workpiece in set-up 1 can similarly be calculated by another connection between the surface $P_{11}$ and the part-holder surface $H_{11}$, as shown in equation (22): 


$$
T_{S 1, P^{4}}=-T_{P^{4}, P_{11}^{4}}+T_{S 1, H 11 S 1}+T_{H 11 S 1, P_{11}^{4}} \cdot
$$

$T_{H 11 S 1, P_{11}^{4}}$ is a link deviation torsor modelling characteristic link between plane $P_{11}$ of the workpiece and the surface $H_{11}$ of the part-holder.

The positioning deviations of the part 4 in set-up $S 1$ are modelled by the torsor $T_{S 1, P^{4}}$, as given in the equation (18) and (22). Thus, it is possible to write a linear system of equations by transforming from the local coordinate system to the global coordinate systems of the shaft $\left(\mathrm{O}_{4} \mathrm{X}_{4} Y_{4} Z_{4}\right)$. It is described by equation (23).

$$
\left\{\begin{array}{l}
\operatorname{lr} x_{4,10 S 1}-l r x_{4,11 S 1}-r x_{4,10}+r x_{4,11}+r x_{4,10 S 1}-r x_{4,11 S 1}=0 \\
l r y_{4,10 S 1}-l r y_{4,11 S 1}-r y_{4,10}+r y_{4,11}+r y_{4,10 S 1}-r y_{4,11 S 1}=0 \\
\text { Ulrz } z_{4,10 S 1}-U l r z_{4,11 S 1}=0 \\
-300 l r y_{4,10 S 1}+350 l r y_{4,11 S 1}+l t x_{4,10 S 1}+300 r y_{4,10}-350 r y_{4,11} \\
-300 r y_{4,10 S 1}+350 r y_{4,11 S 1}-t x_{4,10}+t x_{4,10 S 1}-U l t x_{4,11 S 1}=0 \\
300 l r x_{4,10 S 1}-350 l r x_{4,11 S 1}+l t y_{4,10 S 1}-300 r x_{4,10}+350 r x_{4,11} \\
+300 r x_{4,10 S 1}-350 r x_{4,11 S 1}-t y_{4,10}+t y_{4,10 S 1}-U l t y_{4,11 S 1}=0 \\
-l t z_{4,11 S 1}+t z_{4,11}-t z_{4,11 S 1}+U l t z_{4,10 S 1}=0
\end{array}\right.
$$

Equations (23a) and (23b) are compatibility equations representing overconstraint degrees. The undetermined components (Ulrz $z_{4,10 S 1}$, Ultz $_{4,10 S 1}$, Ultx $_{4,11 S 1}$, Ulty $_{4,11 S 1}$, $\left.U l r z_{4,11 S 1}\right)$ in the link deviation torsor $T_{S 1, P^{4}}$ are eliminated by Gauss-elimination method. Two components $U l r z_{4,10 S 1}$ and $U l r z_{4,11 S 1}$ are equal (cf. equation (22.c)). Thus, their values remain undetermined and will simply be assigned zero value. These components represent the angular position of the shaft in the chuck jaw of the machine. Concerning the determined components $(l r, l t)$, their values depend on the positioning constraints, i.e., non-penetration conditions and positioning functions of each connection. The determination of these components has been presented by Kamali Nejad et al. (2009). Finally, the torsor $T_{S 1, P^{4}}$ modelling the positioning deviation of workpiece in set-up 1 is fully determined by equation (24).

$$
T_{S 1, P^{4}}=\left\{\begin{array}{ll}
l r x_{4,10 S 1}-r x_{4,10}+r x_{4,10 S 1} & -300 l r y_{4,10 S 1}+l t x_{4,10 S 1}+300 r y_{4,10} \\
& -300 r y_{4,10 S 1}-t x_{4,10}+t x_{4,10 S 1} \\
l r y_{4,10 S 1}-r y_{4,10}+r y_{4,10 S 1} & 300 l r x_{4,10 S 1}+l t y_{4,10 S 1}-300 r x_{4,10} \\
& +300 r x_{4,10 S 1}-t y_{4,10}+t y_{4,10 S 1} \\
0 & l t z_{4,11 S 1}-t z_{4,11}+t z_{4,11 S 1}
\end{array}\right\}_{\left(O_{4}, X_{4} Y_{4} Z_{4}\right)}
$$


This method is then applied for each set-up The geometrical deviations of all surfaces of the shaft are modelled by surface deviation torsors, for surface $j$ this torsor will be named $T_{P^{4}, P^{4}}$. For example, the surface deviations of the planar head $P_{1}$ (see Figure 5) of the shaft relative to its nominal position is described by a torsor as shown in equation (25).

$$
T_{P^{4}, P_{1}^{4}}=\left[\begin{array}{ll}
-l r x_{4,10 S 1}-l r x_{4,8 S 2} & 300 l r y_{4,10 S 1}+50 l r y_{4,8 S 2}-l t x_{4,10 S 1} \\
+r x_{4,1}+r x_{4,8}+r x_{4,10} & -l t x_{4,8 S 2}-350 r y_{4,1}-81 r y_{4,8}-300 r y_{4,10} \\
-r x_{4,10 S 1}-r x_{4,8 S 2} & +300 r y_{4,10 S 1}+50 r y_{4,8 S 2}+t x_{4,8} \\
& +t x_{4,10}-t x_{4,10 S 1}-t x_{4,8 S 2} \\
-l r y_{4,10 S 1}-l r y_{4,8 S 2} & -300 l r x_{4,10 S 1}-50 l r x_{4,8 S 2}-l t y_{4,10 S 1} \\
+r y_{4,1}+r y_{4,8}+r y_{4,10} & -l t y_{4,8 S 2}+350 r x_{4,1}+81 r x_{4,8}+300 r x_{4,10} \\
-r y_{4,10 S 1}-r y_{4,8 S 2} & -300 r x_{4,10 S 1}-50 r x_{4,8 S 2}+t y_{4,8} \\
& +t y_{4,10}-t y_{4,10 S 1}-t y_{4,8 S 2} \\
& -l t z_{4,11 S 1}-l t z_{4,9 S 2}+t z_{4,1}+t z_{4,9} \\
& +t z_{4,11}-t z_{4,11 S 1}-t z_{4,9 S 2}
\end{array}\right]_{\left(O_{4}, X_{4} Y_{4} Z_{4}\right)}
$$

In this torsor, the parameters $r x_{4,1}, r x_{4,8}, r x_{4,10}, r y_{4,1}, r y_{4,8}, r y_{4,10}, t z_{4,1}, t z_{4,9}$ and $t z_{4,11}$ represent the machining deviations of the surfaces $\left(P_{1}, P_{9}, P_{11}, C_{8}, C_{10}\right)$ of the shaft and the parameters $r x_{4,10 S 1}, r x_{4,8 S 2}, r y_{4,10 S 1}, t x_{4,10 S 1}, t x_{4,8 S 2}, t y_{4,10 S 1}, t y_{4,8 S 2}, t z_{4,11 S 1}$ and $t z_{4,9 S 2}$ represent the deviations of the fixture surfaces in set-up 1 and set-up 2.

The parameters $l r x_{4,10 S 1}, l r x_{4,8 S 2}, l r y_{4,10 S 1}, l r y_{4,8 S 2}, l t x_{4,10 S 1}, l t x_{4,8 S 2}, l t y_{4,10 S 1}$, lty $y_{4,8 S 2}$, $l t z_{4,11 S 1}$ and $l t z_{4,9 S 2}$ represent the link between the fixture surfaces and the surfaces of workpiece in the set-up 1 and set-up 2.

\subsection{Model of Assembled Part of centrifugal pump}

The manufactured parts of the pump are then assembled according to the selected assembly process. The assembly graph is shown in Figure 6. The geometrical deviations of all surfaces of the pump are described by the torsor in the MAP. For example, the deviations of surface 5 of the impeller (part 5) are calculated by equation (26).

$$
T_{P, P_{5}^{5}}=T_{P, P^{5}}+T_{P^{5}, P_{5}^{5}}
$$

where the surface deviation torsor $T_{P^{5}, P_{5}^{5}}$ is collected by MMP of the impeller.

The part deviation torsor $T_{P, P^{S}}$ models the positioning deviation of the impeller relative to its nominal position in the global coordinate system of product (OXYZ). It is determined by the two connections between the part 4 (the shaft) and part 5 (the impeller). The first connection links the surface 4 of the part 4 and the surface 4 of the part 5. The second one is between the surface 5 of the part 4 and the surface 5 of the part 5 (see Figure 6). The part deviation torsor $T_{P, P^{5}}$ is expressed by equations (27) and (28) from first and second connections, respectively. 


$$
\begin{aligned}
& T_{P, P^{5}}=T_{P, P^{4}}+T_{P^{4}, P_{4}^{4}}+T_{P_{4}^{4}, P_{4}^{s}}-T_{P^{5}, P_{4}^{5}} \\
& T_{P, P^{5}}=T_{P, P^{4}}+T_{P^{4}, P_{5}^{4}}+T_{P_{5}^{4}, P_{5}^{5}}-T_{P^{5}, P_{5}^{5}} .
\end{aligned}
$$

The undetermined components in the link deviation torsor $T_{P_{4}^{4}, P_{4}^{5}}$ and $T_{P_{4}^{4}, P_{6}^{5}}$ are solved by unification method as presented above. The part deviation torsor $T_{P, P^{4}}$ of part 4 of the pump is determined by the same way as $T_{P, P^{5}}$ based on two connections between part 4 and the part 2 (the ring). As shown in Figure 6, the first connection is between the surface 6 of the part 2 and the surface 7 of the part 4 . The second one is between the surface 2 of the part 2 and the surface 6 of the part 4 . The part deviation torsor $T_{P, P^{2}}$ is similarly determined by two connections between part 2 and the part 1 (the back casing) of the pump (see Figure 6).

$$
T_{P, P^{2}}=\left[\begin{array}{ll}
-l r x_{1,6 S 1}+r x_{1,6}+r x_{1,7}-r x_{1,6 S 1} & -35 l r y_{1,6 S 1}-l t x_{1,4 S 1}+35 r y_{1,6}+\frac{45}{2} r y_{1,7} \\
-r x_{2,3}+l r x_{1,7 \rightarrow 2,3} & -35 r y_{1,6 S 1}-\frac{45}{2} r y_{2,3}+t x_{1,4}+t x_{1,7}-t x_{1,4 S 1} \\
l r y_{1,6 S 1}+r y_{1,6}+r y_{1,7}-r y_{1,6 S 1} & 35 l r x_{1,6 S 1}-l t y_{1,4 S 1}-35 r x_{1,6}+\frac{45}{2} r x_{1,7} \\
-r y_{2,3}+l r y_{1,7 \rightarrow 2,3} & +35 r x_{1,6 S 1}+\frac{45}{2} r x_{2,3}+t y_{1,4}+t y_{1,7}-t y_{1,4 S 1}+l t x_{1,7 \rightarrow 2,3} \\
& -t y_{2,3}-\frac{45}{2} l r x_{1,7 \rightarrow 2,3}+l t y_{1,7 \rightarrow 2,3} \\
0 & -l t z_{1,6 S 1}+t z_{1,6}+t z_{1,8}-t z_{1,6 S 1} \\
& -t z_{2,4}+l t z_{1,8 \rightarrow 2,4}
\end{array}\right]_{(O, X Y Z)}
$$

The part deviation torsor $T_{P, P^{1}}$ models the positioning deviations of part 1 (casing back) of the pump relative to its nominal position in the global coordinate system of the pump. In this case, the global coordinate system of the pump is positioned on part 1. Thus, part deviation torsor $T_{P, P^{1}}$ is equal zero. The part deviation torsor $T_{P, P^{2}}$ is calculated and expressed by equation (29).

In this torsor, the parameters $l t x_{1,7 \rightarrow 2,3}, l t y_{1,7 \rightarrow 2,3}$ represent the link between the surface 7 of part 1 and the surfaces 3 of part 2 and lty $_{1,8 \rightarrow 2,4}$ represents the link between the surface 8 of part 1 and the surfaces 4 of part 2 .

In conclusion, the geometrical deviations of all surfaces of the pump relative to its nominal position in the global coordinate system can be modelled according to the selected manufacturing and assembly processes and the associated resources. Thus, the gap deviation torsor between the casing (part 3) and the impeller (part 5) of the pump (see Figure 7) can be calculated based on the GDM by equation (30):

$$
T_{P_{8}^{3}, P_{6}^{5}}=-T_{P, P_{8}^{3}}+T_{P, P_{6}^{5}}
$$


where, $T_{P, P_{8}^{3}}$ is a surface deviation torsor modelling the geometrical deviations of the conic surface $C_{8}$ of the casing in the global coordinate system of the pump.

$T_{P, P_{6}^{5}}$ is a surface deviation torsor modelling the geometrical deviations of the conic surface $C_{6}$ of the impeller of the pump in the global coordinate system.

The torsor $T_{P_{8}^{3}, P_{6}^{5}}$ is described by rotational vector $R$ and translational vector $D$ as shown in equation (5).

The deviation gap between the casing and the impeller of the pump is used to verify a non contact condition between the moving surface of the impeller and the motionless surface of the casing, as shown in equation (31).

$$
\text { Deviation Gap }=\vec{D} \cdot \vec{n} \geq 0
$$

where, $\vec{n}$ is a unit vector along normal direction of the gap.

Figure 6 Assembly graph of the pump (see online version for colours)

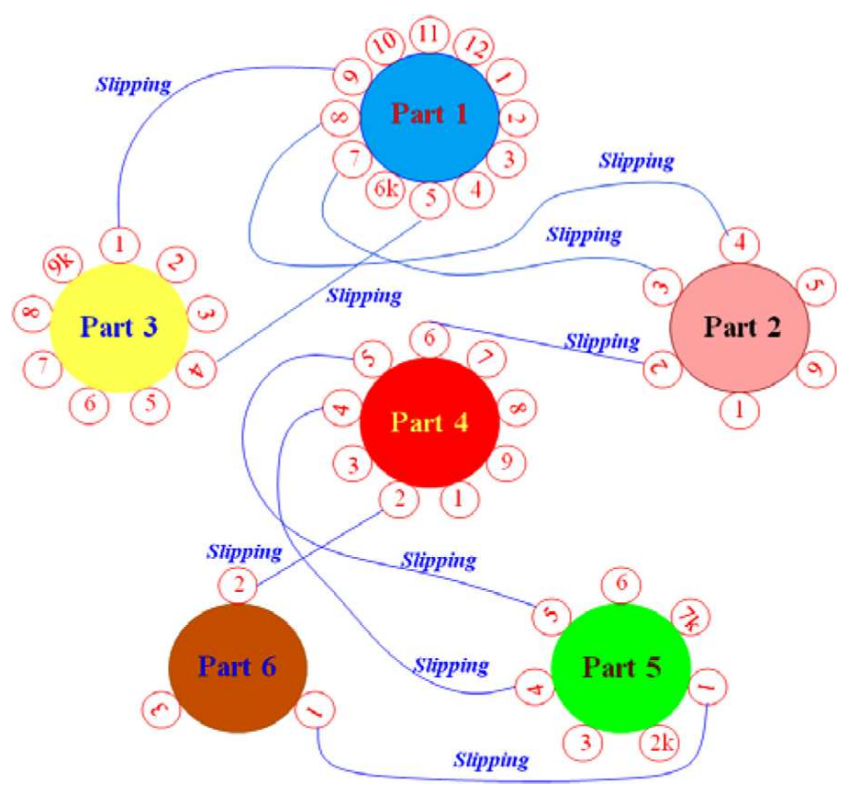

Figure 7 The gap between the casing and the impeller of the pump (see online version for colours)

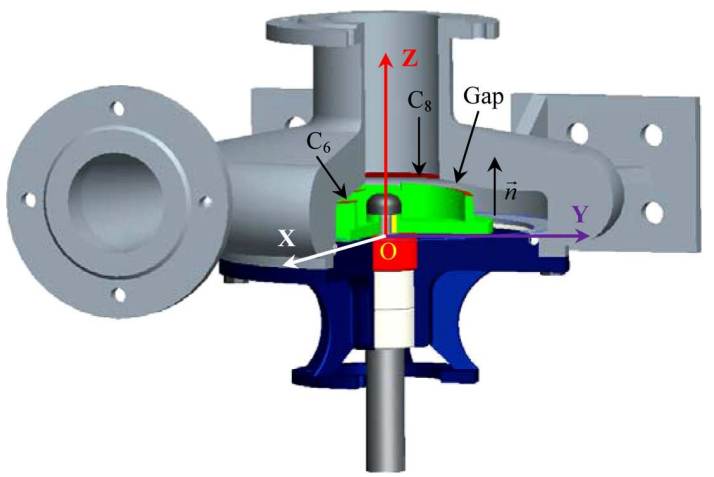


Moreover, this model is also used for analysing assemblability and performance of the pump, such as flow rate, velocity of fluid, pressure and non contact between moving and motionless surface.

\section{Conclusion}

Today, product designers work on a numerical model of the product within a CAx system. This model only represents nominal product information. For the real product, geometrical deviations are generated in each stage of its life cycle. They can make the designed product not to meet fully the requirements of the customers and the users. This paper proposes a model of geometrical deviations of all surfaces of the product consistent with the whole life cycle. During the manufacturing stage, these deviations of surfaces of the manufactured part generated and accumulated by the manufacturing processes are modelled by the MMP. These deviations accumulated once more during the assembly stage are collected in the MAP.

With the proposed model, the product designer can determine the distribution of the geometrical variations of surfaces of the product by the way of Monte-Carlo simulation method. We can then verify the assemblability of the product and take the geometrical deviations of the product into account in the performance simulations. Thus, we can ensure that the product he is designing will have 'real' performances satisfying customers and users.

In future work, we will identify and classify the variation sources that have a strong influence on the performance of the product. This identification will be based on the results of the performance simulations. Further, we could contribute to calculate the variance of the performance of the product during its life cycle. It could lead to optimisation of the design to obtain a robust design.

\section{References}

Bourdet, P. and Clement, A. (1976) 'Controlling a complex surface with a 3 axis measuring machine', Annals of the CIRP, Vol. 25, Manufacturing Technology, pp.359-361.

Bourdet, P., Mathieu, L., Lartigue, C. and Ballu, A. (1996) 'The concept of the small displacement torsor in metrology', Advanced Mathematical Tools in Metrology II, Edited by World Scientific Publishing Company, Series Advances in Mathematics for Applied Sciences, Vol. 40, pp.110-122.

Ceglarek, D. and Shi, J. (1995) 'Dimensional variation reduction for automotive body assembly', Manufacturing Review, Vol. 8, No. 2, pp.139-154.

Dantan, J., Ballu, A. and Mathieu, L. (2008) 'Geometrical product specifications - model for product life cycle', Computer-Aided Design, Vol. 40, pp.493-501.

Ghasempoor, A., Yang, Y. and Xi, F. (2007) 'A geometrical method for three-dimensional modelling of surface finish in grinding', Int. J. Manufacturing Research, Vol. 2, No. 4, pp.389-402.

Hu, S.J. and Stecke, K.E. (2009) 'Analysis of automotive body assembly system configurations for quality and productivity', Int. J. Manufacturing Research, Vol. 4, No. 3, pp.281-305.

Huang, W., Lin, J., Bezdecny, M., Kong, Z. and Ceglarek, D. (2007) 'Stream-of-variation modelling - Part I: a generic three-dimensional variation model for rigid-body assembly in single station assembly processes', Journal of Manufacturing Science and Engineering, Vol. 129, pp.821-831. 
Kamali Nejad, M., Vignat, F. and Villeneuve, F. (2009) 'Simulation of the geometrical defects of manufacturing', The International Journal of Advanced Manufacturing Technology, Vol. 45, Nos. 7-8, pp.631-648.

Mantripragada, R. and Whitney, E.D. (1999) 'Modelling and controlling variation propagation in mechanical assemblies', IEEE Transactions on Robotics and Automation, Vol.15, No. 1, pp.124-140.

Mohanasundararaju, N., Sivasubramanian, R. and Alagumurthi, N. (2008) 'Optimisation of work roll grinding using response surface methodology and evolutionary algorithm', Int. J. Manufacturing Research, Vol. 3, No. 2, pp.236-251.

Ramesh, R., Mannan, M.A. and Poo, A.N. (2000a) 'Error compensation in machine tools - a review - Part I: geometric, cutting-force induced and fixture-dependent errors', International Journal of Machine Tools \& Manufacture, Vol. 40, pp.1235-1256.

Ramesh, R., Mannan, M.A. and Poo, A.N. (2000b) 'Error compensation in machine tools - a review: Part II: thermal errors', International Journal of Machine Tools \& Manufacture, Vol. 40, pp.1257-1284.

Sahoo, P., Barman, T.K. and Routara, B.C. (2008) 'Fractal dimension modelling of surface profile and optimisation in CNC end milling using response surface method', Int. J. Manufacturing Research, Vol. 3, No. 3, pp.360-377.

Shiu, B.W., Ceglarek, D. and Shi, J. (1996) 'Multi-stations sheet metal assembly modelling and diagnostics', Transactions of NAMRI/SME, Vol. XXIV, pp.199-204.

Thangavel, P. and Selladurai, V. (2008) 'An experimental investigation on the effect of turning parameters on surface roughness', Int. J. Manufacturing Research, Vol. 3, No. 3, pp.285-300.

Thiebaut, F. (2001) Contribution à la définition d'un moyen unifié de gestion de la géométrie réaliste basée sur le calcul des lois de comportement des mécanismes, PhD Thesis, ENS de Cachan, France.

Tichadou, S., Kamali Nejad, M., Vignat, F. and Legoff, O. (2007) '3-D manufacturing dispersions: two experimental applications', Proceedings of the 10th CIRP International Seminar on Computer Aided Tolerancing, France.

Tichadou, S., Legoff, O. and Hascoet, J.Y. (2005) '3D Geometrical Manufacturing Simulation: compared approaches between integrated CAD/CAM systems and small displacement torsor models', in Brissaud, D., Bramley, A., Coutellier, D. and McMahon, C. (Eds.): Advances in Integrated Design and Manufacturing in Mechanical Engineering, Springer Netherlands, pp.201-214.

Vignat, F. and Villeneuve, F. (2007) 'Simulation of the manufacturing process, generation of a model of the manufactured parts', in Cunha, P.F. and Maropoulos, P.G. (Eds.): Digital Enterprise Technology, Springer, New York, pp.545-552.

Villeneuve, F. and Vignat, F. (2005) 'Simulation of the manufacturing process (1) Generic resolution of the positioning problem', Proceedings of the 9th CIRP International Seminar on Computer Aided Tolerancing, Tempe, USA, pp.10.

Villeneuve, F., Legoff, O. and Landon, Y. (2001) 'Tolerancing for manufacturing: a three-dimensional model', International Journal of Production Research, Vol. 39, No. 8, pp.1625-1648.

Zhou, S., Huang, Q. and Shi, J. (2003) 'State space modelling of dimensional variation propagation in multistage machining process using differential motion vectors', IEEE Transactions on Robotics and Automation, Vol. 19, No. 2, pp.296-309. 